\title{
The emerging field of nanotoxicology
}

\author{
Christy L. Haynes
}

Published online: 21 July 2010

(C) Springer-Verlag 2010

\begin{abstract}
Although nanoparticles have been intentionally used for centuries, the unique chemical and physical properties of these materials have only been systematically investigated in the last few decades. Since the scientific community came to understand the breadth of new material properties available, it has become increasingly common for nanoparticles to be incorporated into consumer products. In the USA, there are no nanoparticle-specific regulations, and whether or not there should be is still under debate. Either way, it is abundantly clear that nanoscale materials often behave differently from either their bulk or their atomic counterparts and, on the basis of their size, are likely to penetrate biological/ecological systems in novel ways. Thus, the field of nanotoxicology, the study of the adverse affects of nanoscale materials on biological or ecological systems, has emerged.

In the early days of this field (circa 2004), there was a lot of discussion about what needed to be done and not a lot of results. The expansion of the field of nanotoxicology has been fast, and it is clear from the literature in general and this special issue of Analytical and Bioanalytical Chemistry
\end{abstract}

C. L. Haynes $(\bowtie)$

Department of Chemistry, University of Minnesota,

207 Pleasant Street SE,

Minneapolis, MN 55455, USA

e-mail: chaynes@umn.edu that the results are now numerous and exciting. The International Council on Nanotechnology (ICON) keeps a database of publications related to nanomaterial environmental health and safety that reveals interesting trends about the field of nanotoxicology. First, the sheer number of publications has increased drastically from 83 papers in 1999, when nanoscience was already a hot topic, to 154 in 2004, when the first dedicated nanotoxicology conference occurred, to 1,284 papers last year (Fig. 1). Of those, nearly $99 \%$ of the published papers (in 2009) focused on engineered nanoparticles rather than incidental nanoparticles and more than $70 \%$ of the papers reported the use of in vitro assays without any in vivo comparison. I mention these points because they reveal some unique challenges to which analytical and bioanalytical chemists are especially qualified to contribute. Although exposure to incidental nanoparticles, such as those found in air pollution, is certainly more common than exposure to engineered nanoparticles, issues of sampling and characterization of various samples plague studies of incidental nanotoxicology. To complicate this issue further, some studies go to great pains to keep nanoparticles from aggregating or agglomerating, simply because this complicates data interpretation, though the agglomerates/aggregates may, in fact, be the exposurerelevant form of the nanoparticles. Although in vitro assays of nanotoxicity are drastically more common than in vivo assays, for obvious reasons, there is often a lack of correlation between in vitro and in vivo results - this inconsistency calls for the design of better assays with physiologically relevant end points. Another critical component of every nanotoxicology experiment is the use of valid 
Fig. 1 Number of nanotoxicology papers published from 1980 to date

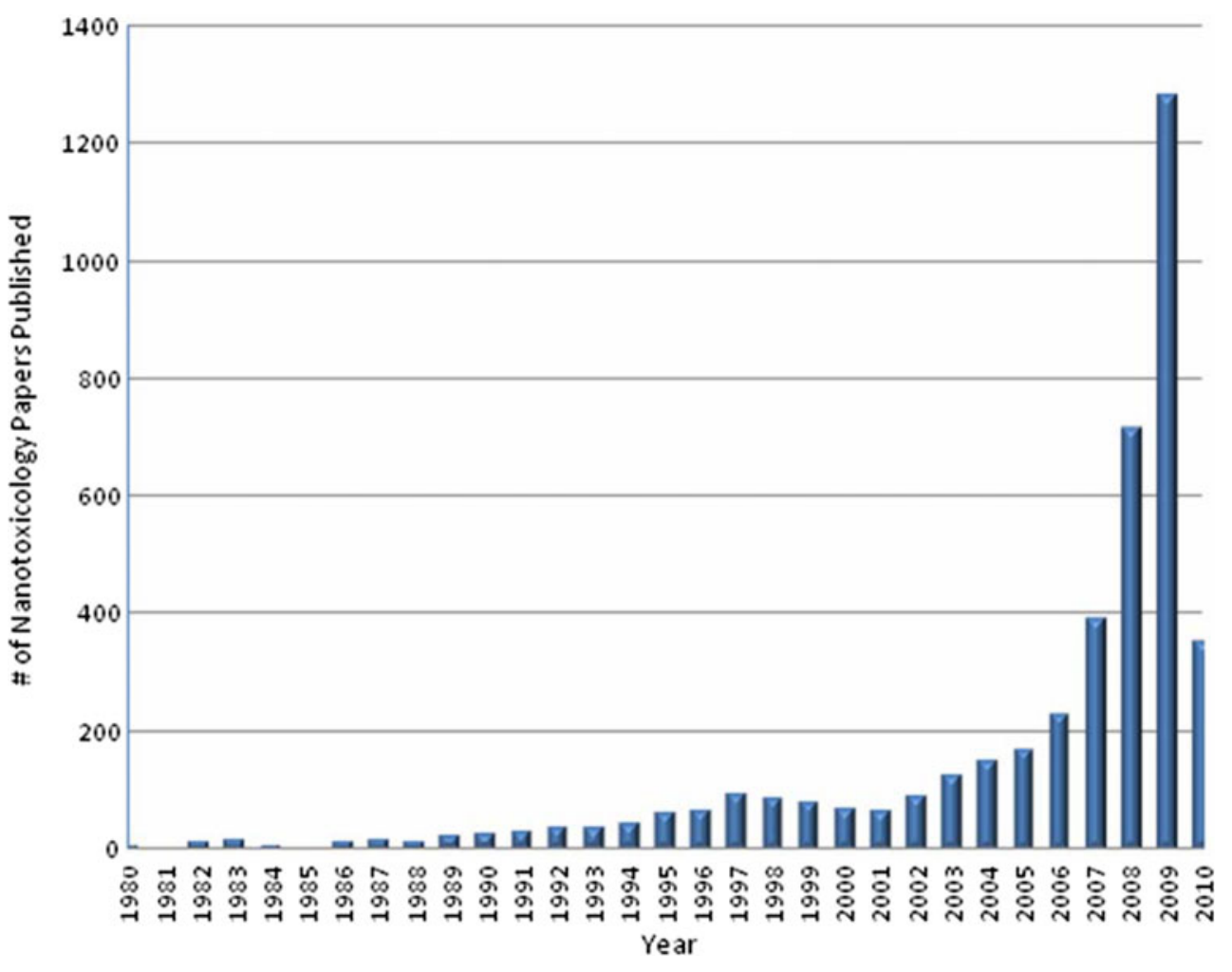

controls. There were some early reports of nanoparticle toxicity in the brain, whereas, in fact, the toxic effect came from the solvent suspending the nanoparticles rather than the nanoparticles themselves. This example demonstrates the need to separate solvent effects from dissolved nanoparticle effects from intact nanoparticle effects - if the nanoparticles themselves are not a problem, then sample cleanup is the main challenge before use. Clearly, the natural strengths of analytical chemists - sampling, assay design, and choice of appropriate controls, among others - are much needed in this emerging field. In addition, this field calls for scientists who are interested in crossing the traditional disciplinary boundaries to ensure that we are asking the right questions and measuring the results to the best of our abilities. Within this issue of Analytical and Bioanalytical Chemistry, you will find some new results, some new approaches, and some detailed reviews in the field of nanotoxicology... but we need more.

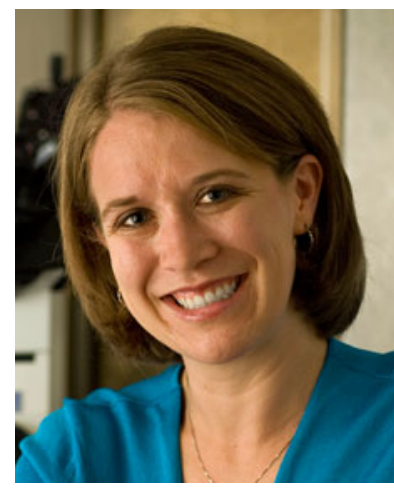

Christy L. Haynes

is Associate Professor of Chemistry at the University of Minnesota. Since arriving in 2005, she has developed a diverse research program that includes single-cell assays for nanotoxicology, fabrication of drug-delivery nanoparticles, monitoring chemical messenger release from immune system cells, and development of surface-enhanced Raman sensing platforms. She is the author or coauthor of 50 scientific publications and serves on the editorial board of multiple journals. During her time as a faculty member, she has received multiple prestigious awards, including the NIH New Innovator, the Searle Scholar Award, the Sloan Fellowship, and a Dreyfus TeacherScholar Award. Her work in the area of nanotoxicology has recently been recognized by the Society for Electroanalytical Chemistry Young Investigator Award as well as the American Chemical Society Division of Analytical Chemistry Findeis Award. 